\title{
Complications After Inadequate Treatment of Nasal Bone Fracture Combined With Septal Fracture: A Case Report
}

\author{
Seung Koo Yang, MD and Seung-No Hong, MD \\ Department of Otorhinolaryngology-Head and Neck Surgery, Seoul National University College of Medicine, \\ Seoul National University Boramae Medical Center, Seoul, Republic of Korea
}

\begin{abstract}
In treating a nasal bone, an incomplete nasal bone reduction can result in nasal deformity, both aesthetic and functional, requiring a secondary operation. A 60-year-old female with a traumatic comminuted nasal bone fracture with septal fracture was initially treated with closed reduction, which later resulted in a saddle nose and a completely occluded nasal cavity. Later, the patient underwent a revision operation of extracorporeal septorhinoplasty with rib cartilage graft, and the postoperative course was uneventful. Herein, we present a case of complications occurring after inadequate treatment of septal fracture. Physicians should thoroughly evaluate the extent of the nasal bone fracture, including the integrity of the nasal septum, and manage accordingly.
\end{abstract}

Keywords: Nasal bone fracture; Septal fracture; Closed reduction; Complication; Rhinoplasty; Extracorporeal septoplasty.

\section{INTRODUCTION}

The nose, being the most protruding anatomical feature on the face, accounts for the nasal bone fracture to be the most common type of facial bone fracture. ${ }^{1)}$ Nasal bone fracture can be classified into different types, depending on the extent of the fracture. The type, which ultimately reflects the severity of the fracture, affects surgeons in choosing the surgical approach with the nasal bone reduction; a closed approach is recommended for simple types of fracture while an open approach is suggested for more severe types of fracture. Whether it be closed or open reduction, several complications may occur after the reduction of nasal bone fracture: nasal deformity, septal deviation, nasal obstruction, olfactory disturbance, and diplopia. ${ }^{2)}$ Depending on the type and severity of the complication, a secondary surgery may be required to address the problem, and the rate of postreduction deformity undergoing subsequent septorhinoplasty was reported

Received: March 19, 2021 Revised: April 12, 2021

Accepted: April 26, 2021

Address for correspondence: Seung-No Hong, MD, Department of Otorhinolaryngology-Head and Neck Surgery, Seoul National University College of Medicine, Seoul National University Boramae Medical Center, 20 Boramae-ro 5-gil, Dongjak-gu, Seoul 07061, Republic of Korea

Tel: +82-2-870-2114, Fax: +82-2-870-3866, E-mail: seuno77@gmail.com

This is an Open Access article distributed under the terms of the Creative Commons Attribution Non-Commercial License (https://creativecommons.org/licenses/bync/4.0) which permits unrestricted non-commercial use, distribution, and reproduction in any medium, provided the original work is properly cited. to range from $14 \%$ to $50 \%{ }^{3)}$

Here, we describe a case in which a closed reduction of bilateral comminuted nasal bone fracture with septal fracture resulted in a saddle nose and a completely obstructed nasal cavity, calling for a secondary surgery of extracorporeal septoplasty (ECS). Furthermore, the importance of assessing and managing the septal fracture is discussed based on the literature.

\section{CASE REPORT}

A 60-year-old female fell down the stairs and visited the emergency room on November 28, 2017. She had lacerations on both of her lower eyelids $(2.5 \mathrm{~cm}$ each) and across the dorsum $(3 \mathrm{~cm})$. Face skull bone CT showed comminuted nasal bone fracture on both sides, extending to septum and right maxilla frontal process (Fig. 1). The patient was referred to the department of dental surgery at first and six days later on December 4, 2017, closed nasal bone reduction, which was limited to nasal bone only, was performed, and nasal aqua splint was applied afterwards.

Three months later on March 30, 2018, the patient visited the ENT department for the first time with a chief complaint of severe nasal obstruction. Upon examination, a saddle nose was identified, and the left nasal cavity was completely obliterated due to the severe deviation of the nasal septum to the left, and the right nasal cavity was visible from the left nostril 
via the septal perforation (Fig. 2). A CT scan reflected the physical finding, showing depressed nose and the obliteration of the left nasal cavity with severe deviation of nasal septum (Fig. 3). With the assessment of nose deformity after nasal bone reduction, the patient underwent a revision surgery of extracorporeal septorhinoplasty with rib cartilage graft on April 11, 2018.

During the surgery, a $4 \mathrm{~cm}$-long-segment of sixth rib cartilage, along with perichondrium, was harvested from the right side. Incision was made from the left nasal cavity inlet, and mucosal dissection, with the elevation of the septal mucosa, was performed. Septal mucosa was repositioned to be sutured with the residual right septal mucosa at the caudal end of the nose. The entire septal cartilage was dislocated and taken out. After removing redundant cartilage and fracture lines, the septal cartilage was designed into a new L-strut shape. Rib cartilage was carved and attached to two perpendicular sides of septal cartilage, functioning as bilateral extension spreader grafts on one side and a columellar strut on the other side (Fig. 4). A layer of rib cartilage, reinforced by another layer of perichondrium, was used as a dorsal only graft. The L-strut shaped cartilage was inserted back into its original location, and the left septal mucosa was not sutured to the residual left septal mucosa at the caudal end, so that it could be healed by secondary intension. After the surgery, the saddle nose was corrected, and the left nasal cavity was well reconstructed (Fig. 5). The patient was discharged four days after the surgery without any postoperative complication. At 2-monthfollow-up, the patient visited without any complaint, and the corrected septum and the left nasal cavity maintained its patency (Fig. 6).

The case report was approved by the Ethics Committee of the Seoul National University Boramae Medical Center. Written informed consent was obtained from the patient.
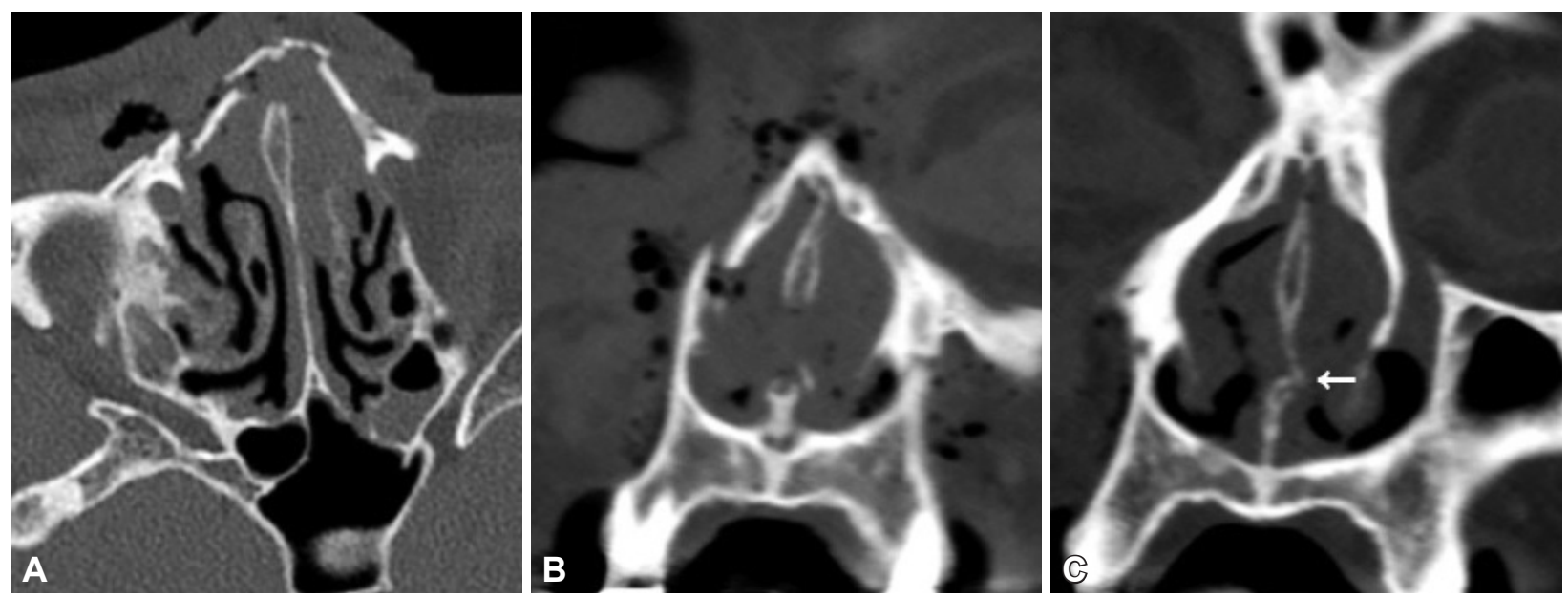

Fig. 1. Initial CT after trauma (A: axial, B, C: coronal). Bilateral comminuted nasal bone fracture, extending to frontal process of right maxilla and nasal septum (arrow), is evident.
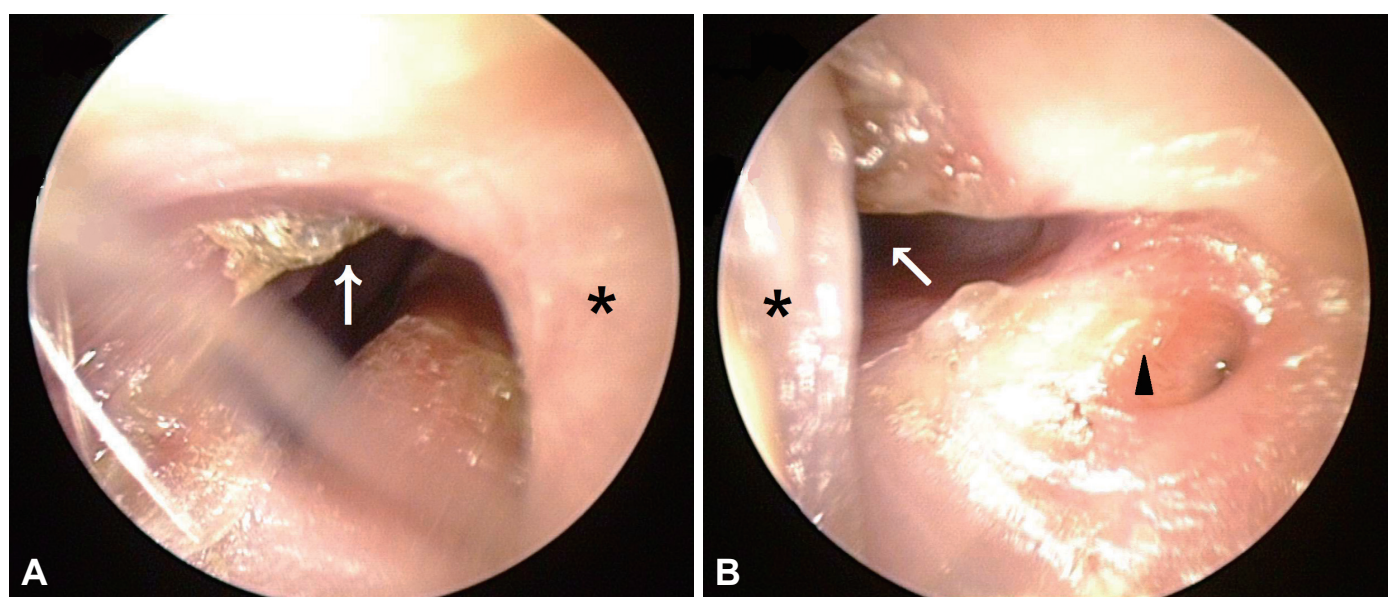

Fig. 2. Endoscopic finding of the nasal cavity after the initial reduction. A: Endoscopy of right nasal cavity with residual anterior septum (asterisk). B: Endoscopy of left nasal cavity (arrowhead), which is completely obliterated. Right nasal cavity (arrow) is visualized from the left nostril via the septal perforation. 


\section{DISCUSSION}

\section{Inadequate reduction of nasal bone fracture}

Although complications of nasal bone fracture itself had been widely studied, complications of the management of nasal bone fracture had not been explored much. Potential
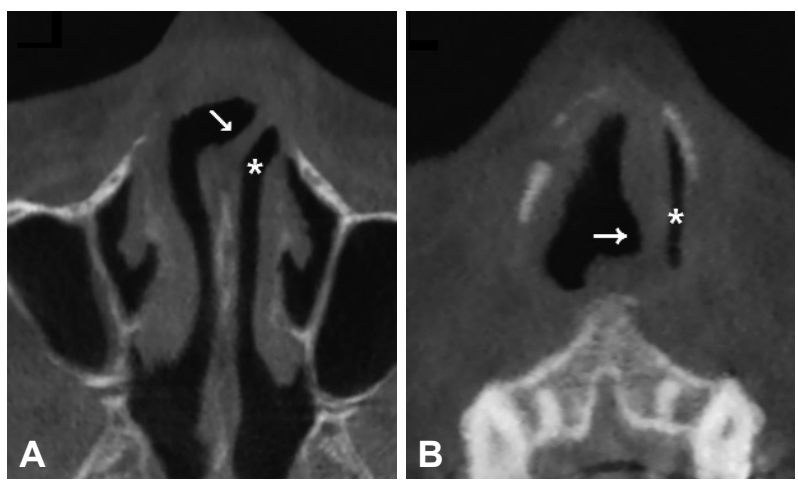

Fig. 3. Pre-operative CT (A: axial, B: coronal). The left nasal cavity is totally blocked (asterisk). Severely deviated nasal septum (arrow). complications after nasal bone reduction include obstructed breathing, infection, recurrent deformity, inadequate correction, asymmetry, patient dissatisfaction, and olfactory disturbances. ${ }^{14)}$ The rate of complication after nasal bone reduction remains high, ranging from $9 \%$ to $50 \%{ }^{5)}$ As a result, patient satisfaction and revision rates after closed reduction range from $33 \%$ to $85 \%$ and $15 \%$ to $50 \%$, respectively. ${ }^{67)}$ The relatively high rate of revision rate after closed reduction often originates from unrecognized injuries of the nasal septum, upper, lower lateral cartilages, and the keystone area, which are not amenable to closed reduction. Incomplete closed reductions often result in persistent C-shaped fractures involving the bony and cartilaginous septum, which often occur in displaced bony fractures. ${ }^{8}$

\section{Importance of assessing nasal septum}

Over the past several decades, the role of septal fracture in nasal bone fracture management has been emphasized, since the most important step in determining the aesthetic, func-
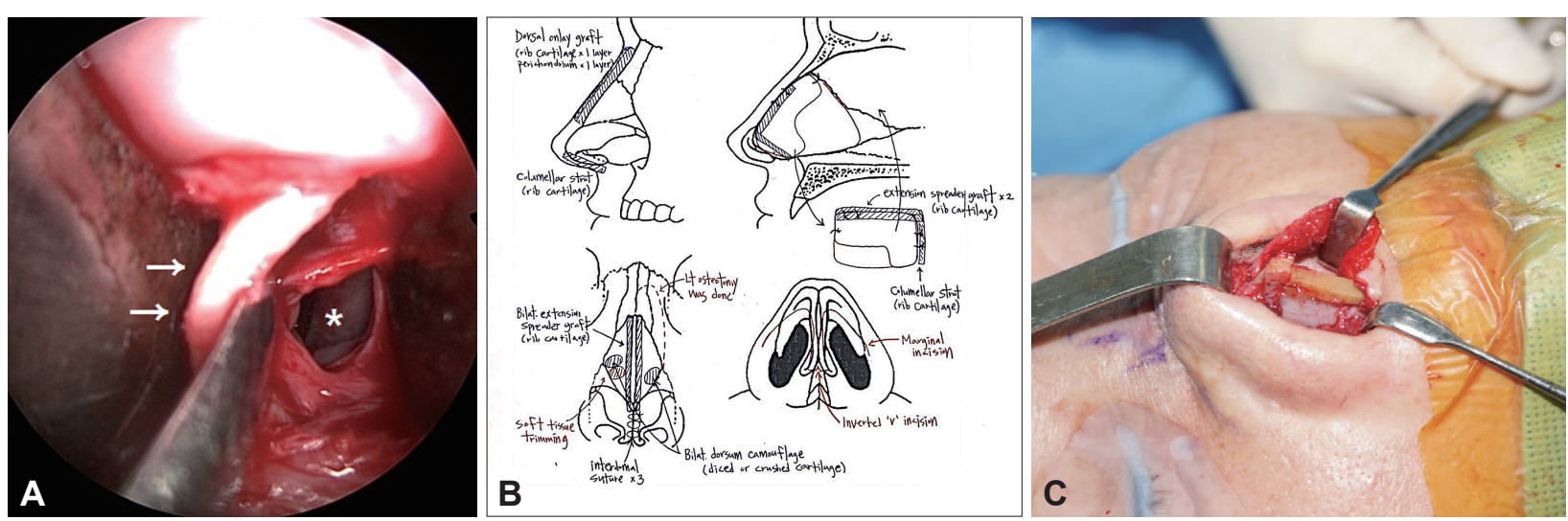

Fig. 4. Operation findings. A: Intra-operative photo of the septal cartilage (arrows). Visualization of the left nasal cavity after dissection (asterisk). B: Rhinoplasty worksheet describing the design of neoseptum with rib cartilage. C: Intraoperative photo of the anterior septal angle reconstructed by columellar strut and extended spreader graft with rib cartilage.
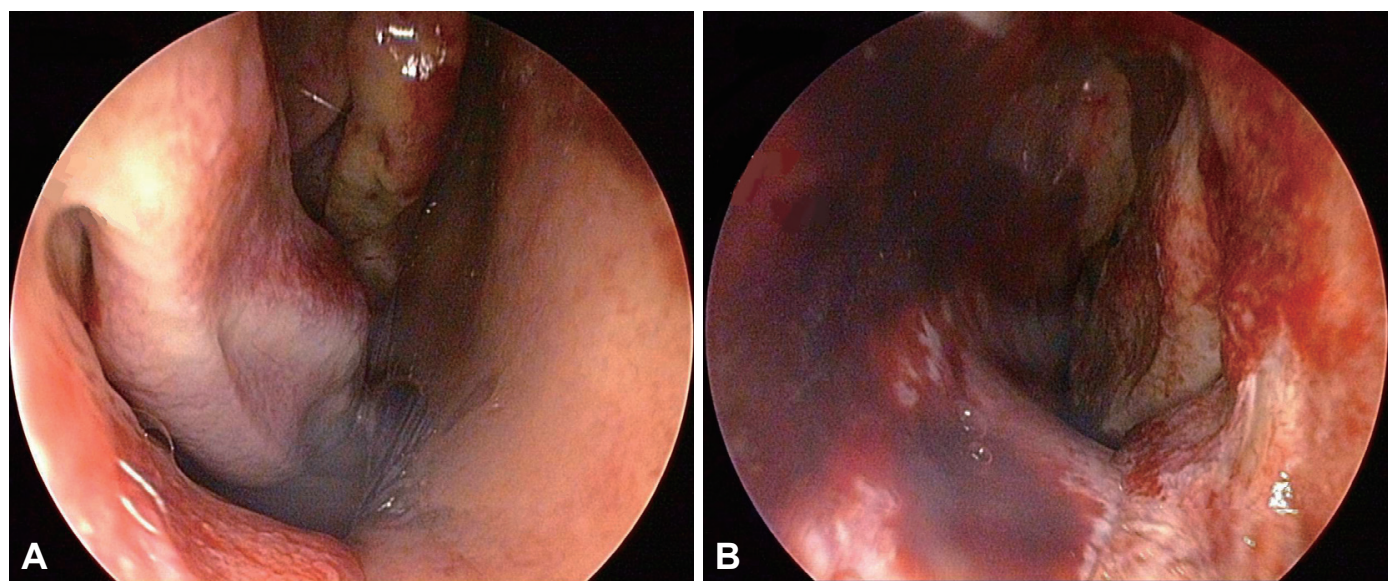

Fig. 5. Post-operative endoscopy (A: right, B: left) showing the correction of the deformities, allowing the visualization of both nasal cavities. 

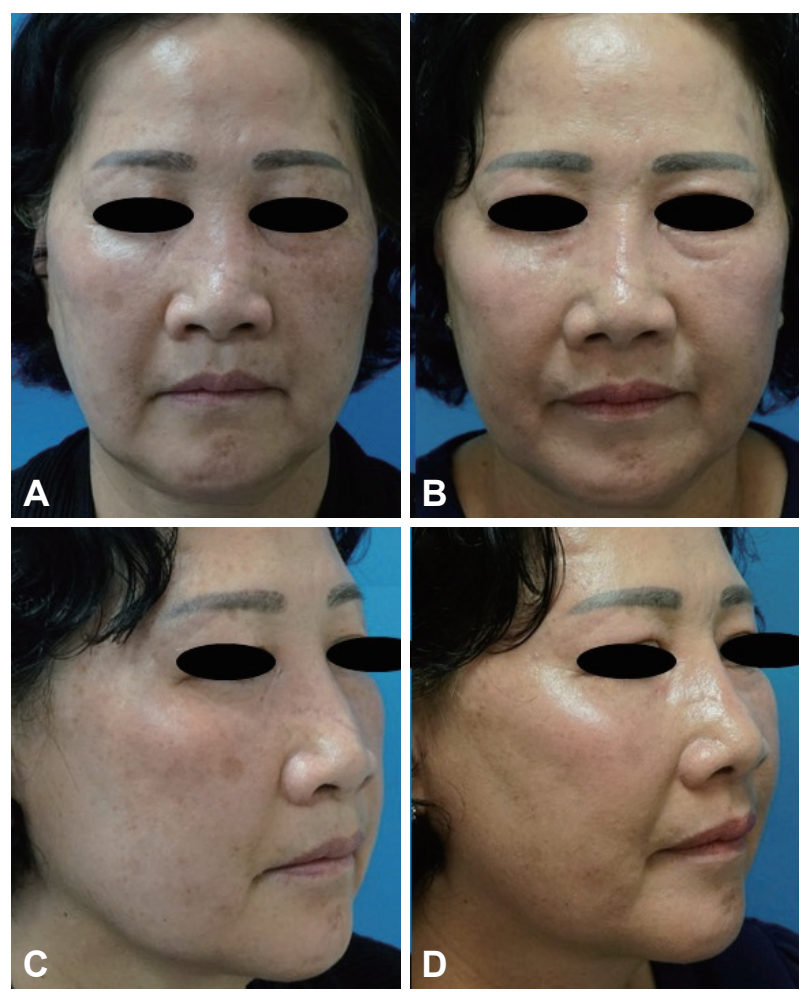

Fig. 6. Pre-operative and post-operative facial photos (A, C: pre, B, $\mathrm{D}$ : post) showing the correction of the saddle nose.

tional outcome of nasal bone fractures is the assessment of the nasal septum. ${ }^{9)}$ The importance of septal assessment lies in the fact that the distortion of septal fracture progresses with time due to the release of locked internal stress system, and fibrosis of healing tissue results in nasal valve deformity and nasal tip imbalance, causing nasal cavity obstruction. ${ }^{10)}$ Even in cases of septal reduction may result in functional deformity after closed reduction, since reduction of anterior portion of septum leaves a dislocated posterior septum intact. ${ }^{11)}$ Previous reports show the rate of concomitant septal fracture in nasal bone fracture patients to be ranging from $46.9 \%$ to $89.7 \%$, and we wonder whether septal assessment was thoroughly done in majority of these patients, considering the rate of complication after closed reduction of the nasal bone fracture. Even a simple physical examination may indirectly hint the possibility of septal fracture, since mucosal tearing was found to be statistically significant for concomitant septal fracture. ${ }^{12)}$ In our case, although the patient was presented with nasal bone fracture involving septal fracture, only closed reduction was performed initially, which ultimately resulted in severe septal deviation and saddle nose, calling for secondary surgery.

\section{Management of septal fracture}

A variety of methods in managing the nasal septum exists, ranging from closed approach with intranasal splints to open approach. ${ }^{3)}$ Severe deviation of the septum may call for ECS, which involves the removal of native deformed septum and the replacement with a reconstructed neoseptum. Literatures show that ECS results in better functional outcome than traditional in situ septorhinoplasty for patients with severe deformities. ${ }^{13)}$ ECS is often used for correcting a severe deviation of nasal septum and is reported to be the most commonly used approach in comminuted fracture with saddle nose deformity. In a case of traumatic comminuted and displaced nasal fracture, ${ }^{8)}$ the patient was initially treated with closed fracture reduction and came back three months after the reduction, complaining of nasal obstruction, headache, and posttraumatic nasal deformity. Upon physical examination, a scoliotic nose and an S-shaped septal deviation were observed, and external rhinoplasty was performed.

\section{Fracture-specific treatment}

An accurate assessment of the extent of the fracture is crucial, and the type of surgical approach should be considered with care to prevent complications that could ultimately lead to a secondary surgery. In other words, understanding different types of fracture is a prerequisite to an appropriate choice of surgical approach. Closed reduction is often used for managing simple nasal bone fractures, while open reduction, possibly in conjunction with rhinoplasty, is used where more precise assessment and reduction are needed. Several authors have reported algorithms in managing the nasal bone fracture according to the extent of the fracture, ${ }^{3) 1314}$ and when the management of nasal bone fracture was determined after thorough evaluation of the extent of the injury, there was no statistical difference between the results of a closed reduction and open reduction of the nasal bone fracture, in terms of patient satisfaction scores, surgeon evaluation scores, and revision rates. ${ }^{14)}$ Revision operation may be avoided if the extent of the nasal bone fracture was thoroughly evaluated in the first place.

In summary, the management of the nasal bone fracture has to be carefully evaluated and carried out depending on the type of fracture, as the complication of initial reduction may result in a secondary surgery, which is often more invasive. ENT specialists should be aware of the extent of the nasal bone fracture and carry out the management accordingly.

\section{Conflicts of Interest}

The authors have no potential conflicts of interest to disclose.

\section{Author Contributions}

Conceptualization: all authors. Formal analysis: Seung-No Hong. Investigation: Seung-No Hong. Resources: Seung-No Hong. Supervision: SeungNo Hong. Writing — original draft: Seung Koo Yang. Writing—review \& editing: Seung-No Hong. 


\section{ORCID iDs}

Seung Koo Yang https://orcid.org/0000-0003-3519-7395

Seung-No Hong https://orcid.org/0000-0002-5759-9929

\section{Funding Statement}

None

\section{REFERENCES}

1) Kang BH, Kang HS, Han JJ, Jung S, Park HJ, Oh HK, et al. A retrospective clinical investigation for the effectiveness of closed reduction on nasal bone fracture. Maxillofac Plast Reconstr Surg 2019; 41(1):53.

2) Hwang K, Yeom SH, Hwang SH. Complications of nasal bone fractures. J Craniofac Surg 2017;28(3):803-5.

3) Rohrich RJ, Adams WP Jr. Nasal fracture management: minimizing secondary nasal deformities. Plast Reconstr Surg 2000;106(2):266-73.

4) Hatef DA, Meaike JD, Hollier LH. Closed reduction of nasal fracture. In: Anh Tran T, Panthaki Z, Hoballah J, Thaller S, editors. Operative dictations in plastic and reconstructive surgery. Cham: Springer;2017. p.277-8.

5) Kim J, Jung HJ, Shim WS. Corrective septorhinoplasty in acute nasal bone fractures. Clin Exp Otorhinolaryngol 2018;11(1):46-51.

6) Arnold MA, Yanik SC, Suryadevara AC. Septal fractures predict poor outcomes after closed nasal reduction: retrospective review and survey. Laryngoscope 2019;129(8):1784-90.

7) Yi JS, Kim MJ, Jang YJ. An Asian perspective on improving outcomes for nasal bone fractures by establishing specific treatment options. Clin Otolaryngol 2017;42(1):46-52.

8) Davis RE, Chu E. Complex nasal fractures in the adult-a changing management philosophy. Facial Plast Surg 2015;31(3):201-15.

9) Verwoerd CD. Present day treatment of nasal fractures: closed versus open reduction. Facial Plast Surg 1992;8(4):220-3.

10) Ridder GJ, Boedeker CC, Fradis M, Schipper J. Technique and timing for closed reduction of isolated nasal fractures: a retrospective study. Ear Nose Throat J 2002;81(1):49-54.

11) Harrison DH. Nasal injuries: their pathogenesis and treatment. $\mathrm{Br} J$ Plast Surg 1979;32(1):57-64.

12) Rhee SC, Kim YK, Cha JH, Kang SR, Park HS. Septal fracture in simple nasal bone fracture. Plast Reconstr Surg 2004;113(1):45-52.

13) Andrades P, Pereira N, Rodriguez D, Borel C, Hernandez R, Villalobos R. A five-year retrospective cohort study analyzing factors influencing complications after nasal trauma. Craniomaxillofac Trauma Reconstr 2019;12(3):175-82.

14) Ondik MP, Lipinski L, Dezfoli S, Fedok FG. The treatment of nasal fractures: a changing paradigm. Arch Facial Plast Surg 2009;11(5): 296-302. 